\title{
L'empirisme idéologique de Dominique Gentil
}

Jean-Pierre Jacob

\section{CpenEdition}

Journals

Édition électronique

URL : http://journals.openedition.org/apad/495

DOI : 10.4000/apad.495

ISSN : 1950-6929

Éditeur

LIT Verlag

Édition imprimée

Date de publication : 1 juin 1999

\section{Référence électronique}

Jean-Pierre Jacob, «L'empirisme idéologique de Dominique Gentil », Bulletin de I'APAD [En ligne], 17

1999, mis en ligne le 04 octobre 2006, consulté le 08 septembre 2020. URL : http://

journals.openedition.org/apad/495; DOI : https://doi.org/10.4000/apad.495

Ce document a été généré automatiquement le 8 septembre 2020.

Bulletin de l'APAD 


\title{
L'empirisme idéologique de Dominique Gentil
}

\author{
Jean-Pierre Jacob
}

1 Le but de l'article (Jacob 1998a) que D. Gentil stigmatise si durement était de souligner l'importance de l'étude des normes, des systèmes de valeur, de la syntaxe politique sous-jacente aux innovations institutionnelles proposées dans le cadre des réformes de l'administration locale. Il me paraissait utile de rappeler, pour quelques collègues qui travaillent sur le thème et privilégient, selon l'excellente tradition apadienne, les études de terrain, les éléments de philosophie politique et les sources d'inspiration théorique auxquels les décideurs doivent faire référence lorsqu'ils produisent une politique dans le domaine de la décentralisation administrative et politique. Mon hypothèse est que les innovations institutionnelles largement imposées de l'extérieur mais mises en œuvre par les Etats africains et donc renégociées par eux, le sont sur la base d'une perception par les décideurs d'un possible conflit d'intérêt entre leurs "acquis" en matière de gouvernance et l'ouverture (difficilement contrôlable à leurs yeux) du champ des possibles dans les rapports entre "le haut et le bas" que proposent les réformes, dans les domaines des relations politiques, des marges de manœuvre et des opportunismes dorénavant accessibles aux différents groupes sociaux, des questionnements socialement légitimes pouvant être formulés à propos de la réalité...

2 En l'occurrence, mon article se fonde sur une distinction - qui n'est pas entièrement explicite dans le texte et le rend du coup incomplet - entre deux interprétations opposées de ce que signifie être un citoyen actif en philosophie du droit.

3 La première interprétation est libérale, individualiste et instrumentalisante et remonte à Locke, la seconde est communautarienne et éthique et s'inspire d'Aristote et de la cité grecque (voir Habermas 1998, Sartori 1987). La première interprétation voit les individus demeurer fondamentalement à l'écart de toutes les formes étatiques, lui apportant des contributions spécifiques (le vote, les impôts) en échange de services sociaux. La seconde considère les citoyens comme intégrés dans la cité politique comme les parties d'un tout, et développant leur identité sociale seulement à travers le cadre des institutions. Dans le premier cas, la liberté du citoyen est vue comme une liberté 
conquise "contre" l'emprise de l'Etat, dans l'autre, sa liberté s'exprime dans sa pleine participation aux décisions politiques. Dans un cas, il existe une forte séparation des registres public et privé que le système représentatif contribue à fonder. Dans l'autre c'est le système de la démocratie directe où le citoyen s'identifie de plein gré aux intérêts de la communauté et où la sphère privée est synonyme de "déprivation" (avoir une vie privée, c'est avoir une vie "déprivée" d'accès à la vie publique, c'est donc être "incomplet").

4 Les réformes des collectivités territoriales en Afrique de l'Ouest sont déterminées par ces sources d'inspiration théoriques, et les pays se voient présenter l'alternative suivante : appliquer la version libérale et suivre à la lettre un agenda largement imposé, ou tenter de trouver un modèle de réforme plus holiste, plus communautarien, là où les décideurs n'acceptent pas une décentralisation "clés en main" ${ }^{1}$. Dans le premier cas, la décentralisation est prise "classiquement" dans un grand ensemble de réformes qui concernent l'Etat de droit, la mise en place d'une démocratie représentative à tous les échelons, l'appui à l'émergence de la société civile (comme concept opposé à l'économie administrée) dans la conduite des tâches productives. Dans le second cas, ce n'est évidemment pas le modèle de la cité grecque qui est promu (en admettant que les Etats ou les populations en veuillent, sa faisabilité est douteuse dès qu'on dépasse un certain nombre de citoyens ${ }^{2}$ ) mais toutes sortes de tentatives ont lieu pour tenter d'euphémiser les effets anticipés d'une démocratie représentative considérée comme "non adaptée" aux manières de faire de la politique et du développement (notamment en milieu rural, voir les débats sur ce thème et les solutions trouvées dans des pays comme le Mali, le Burkina, le Ghana ou l'Ouganda).

5 Ce que mon article traite par contre de manière approfondie, c'est la nature des systèmes politiques établis et rodés dans la durée - cf. les répertoires distingués, Jacob, 1998a: 122-123 - et l'hypothèse selon laquelle les innovations institutionnelles proposées avec les réformes apparaissent comme autant de menaces potentielles pour ces systèmes. Pour résumer mes propos, je dirai que la voie libérale (avec son insistance sur la division du travail public/privé et ses aspects "trade off") peut être perçue comme donnant les arguments nécessaires aux citoyens pour remettre en cause leur mobilisation permanente dans le cadre de la constitution des biens publics ou sociaux. Quant à la voie communautarienne, elle peut être interprétée comme facilitant l'accès à des tribunes publiques pour des minorités politiques et/ou ethniques "intenses", tentées de se servir du tremplin des collectivités territoriales pour réaliser leur autarcie propre, sous couvert de gestion "d'affaires locales", remettant en cause les représentations dominantes de "l'unité de la nation". Bien entendu, les capacités d'anticipation des décideurs et les marges de manœuvre qui leur sont octroyées dans la négociation leur permettent de tenter d'euphémiser, selon les pays, ces dangers potentiels, traités notamment à partir d'une analyse spécifique du rôle des élites politiques locales (voir sur le sujet, Jacob 1998b). Il y faut cependant du temps, ce qui explique la lenteur actuelle dans la mise en œuvre des réformes, voire même les blocages, certains pays s'installant dans un état de "pré-décentralisation" prolongé (Bénin, Tchad).

6 Par ailleurs, et malgré les craintes qu'elles suscitent pour les systèmes de gouvernance établis, on constate une utilisation rhétorique de ces sources d'inspiration théorique (et notamment de la voie holiste, présentée comme voie "originale") mobilisées (dans les politiques de communication par exemple), tant pour s'attirer les bonnes grâces des 
bai1leurs et tenter de séduire les populations que pour chercher à disqualifier des expériences qui peuvent apparaître comme concurrentes. Ainsi la décentralisation est-elle présentée au Mali ou au Burkina, comme une opportunité majeure de créer des structures à la portée des investissements politiques du citoyen de base, une chance d'assister, comme on le dit au Mali, "au retour du pouvoir à la maison" (mara ka seki so), une possibilité de pouvoir enfin parler du gouvernement "à la première personne du pluriel". Bien entendu, ce holisme est présenté comme s'accommodant difficilement d'autres expériences "totales" ${ }^{3}$, opérant dans les mêmes domaines que les structures décentralisées et "s'approvisionnant", de manière concurrentielle, aux mêmes sources physiques et financières que lui. Je veux bien entendu parler de l'ensemble des expériences de développement local (gestion de terroir, développement rural intégré, fonds d'investissements locaux et autres tentatives sectorielles...) soutenues par les différentes coopérations, qui ont toutes développé -mais chacune avec ses instruments, ses méthodes et ses échelles d'intervention - leurs systèmes négociés (participatifs) d'appuis aux populations, parfois depuis de nombreuses années, dans les domaines de la production tant de biens privés que de biens publics (écoles, dispensaires) ${ }^{4}$.

7 Les objectifs de mon article étaient de rappeler ces points. Il jetait les bases d'un projet - sûrement trop ambitieux - d'anthropologie des politiques et des décideurs. Je soulignais que l'étude des réformes de décentralisation exige à la fois à des analyses rétrospectives sur la nature et l'action de l'Etat (voir sur le sujet Slater 1990), les systèmes de relais habituels entre le haut et le bas, et les problèmes politiques internes aux différents pays concernés, et des analyses prospectives sur le potentiel d'allocation des technologies institutionnelles proposées et que c'était à ces conditions qu'on pouvait saisir les enjeux de la décentralisation et les pratiques développées par chaque Etat pour y faire face (l'accent mis, selon les pays, sur les exigences de "cohérence verticale" ou de "cohérence horizontale" renvoyant à des choix différents en matière de techniques représentatives par exemple).

En gros, Dominique Gentil me dit que de tels objectifs sont sans intérêt ("abstraits" dans sa terminologie), et que ma bibliographie est trop fournie. C'est son droit. Ce qui l'est moins c'est de me reprocher de ne pas avoir écrit l'article que je n'ai jamais eu l'intention d'écrire, et ne pas parler du peuple là où j'avais surtout l'intention de parler des élites et des alternatives qui se posent à elles dans la production des réformes.

9 Une fois précisée ma position théorique et donc éclaircis, je l'espère, quelques malentendus de lecture, j'aimerais répondre à ce qui constitue, me semble-t-il, l'essentiel de sa critique, en reprenant le problème des rapports entre développement local et décentralisation, et en dévoilant mieux que je ne l'ai fait dans mon texte - qui n'était pas d'abord écrit pour cela - les principes qui peuvent guider la réflexion normative et, au-delà, ma vision de la coopération au développement.

Les problèmes que posent au fond Gentil sont ceux de savoir quelles sont les définitions possibles du développement local et quels sont les choix institutionnels qui doivent être faits pour l'appuyer. L'intervention des Etats (et des gouvernements locaux) est-elle nécessaire ou le soutien à des "communautés" suffit-il ? Gentil est pour la seconde solution, probablement parce que sa longue expérience l'a conduit à une sorte de "pessimisme méthodologique" (Chauveau) qui oriente sa pratique théorique: empirisme radical, pragmatisme éprouvé, démarche itérative et cumulative, populisme modéré, anthropologie "métisse" (Le Roy). Reconnaissons-le, ce sont bien les anthropologues et les politologues de "terrain" qui nourrissent sa réflexion, les 
chercheurs qui (à l'APAD et ailleurs) ont montré que les interventions top-down sont vouées au dépeçage, à la dissolution dans la multitude des stratégies d'acteurs, et que l'Afrique politique est l'Afrique des factions, de l'Etat absent ou dégradé au point de n'être plus "qu'un groupe comme un autre". Par contre, Gentil ne s'inspire de personne lorsque, sur ces bases, il se lance dans une épistémologie congruente à la légitimitation du type d'action qu'il soutient, définissant ce qui est la "science" (les études précitées) et ce qui ne l'est pas (mon article) et circonscrivant l'objet "développement local" à l'aune de sa pratique.

11 Les hommes impliqués dans l'action font leur la proposition hégélienne selon laquelle "tout ce qui est réel est rationnel". Ils se divisent ensuite sur son interprétation, entre ceux qui considèrent que le "juste" c'est ce qui existe - c'est la position de D.

Gentil pour lequel le rationnel doit s'adapter à la réalité - et ceux qui pensent que le "juste" c'est ce qui pourrait exister. Je pense qu'en matière de politiques de développement et de politiques publiques en général, on ne peut se contenter d'un raisonnement pragmatique sur les expériences acquises, la réalité doit être constamment recadrée par la recherche prospective de normes, de théories, et se soumettre donc, parfois, au rationnel. Quelques réflexions supplémentaires me permettront de justifier cette position.

13 1) Je crois que la valeur exemplaire des expériences actuelles de développement local est limitée. Il y a un biais métonymique à insister, comme le fait Gentil, sur la continuité entre les problèmes des populations et les solutions proposées dans ce domaine. Le bon exercice du développement local suppose à la fois des sites privilégiés, une reformulation "ad hoc" des demandes des bénéficiaires (même dans le cadre relativement non contraignant des fonds d'investissement locaux), des subventions pérennes, des pratiques "expérimentales" de participation non reproductibles à grande échelle.

14 2) Je ne suis pas sûr que l'output organisationnelle plus visible de l'ensemble de ces expériences suffise, lorsqu'il est agrégé, à constituer une société. Ces centaines de milliers de "membres", "cotisants", "sociétaires" constituant des "collectifs privés" à propos desquels Gentil s'enthousiasme, représentent des entreprises respectables et remplissent des fonctions que l'Etat n'assume pas, ne pourrait ni ne devrait assumer, notamment dans le domaine de l'amélioration du bien-être social et économique, mais ils sont incapables, par définition, de poser les problèmes généraux d'équité sociale, d'identité nationale et de cadre politique global dans lequel doivent se prendre les décisions. Au pire, ils renforcent les "patriotismes locaux" et l'Afrique des factions évoqué plus haut. J.-P. Elong Mbassi pose le problème de manière intéressante lorsqu'il remarque que: "...Les projets de développement local sollicitent la capacité d'auto-financement des populations à travers les cotisations alors que le mode de prélèvement des autorités locales est la fiscalité. Cette perpétuation de la logique de la cotisation axée sur des relations interpersonnelles et le bénéfice direct du cotisant soulève en effet la question du développement de la citoyenneté en milieu rural. Cette crise de la citoyenneté dans pratiquement tous les pays africains, malgré la démocratisation, pose en termes critiques le problème de la mobilisation pour le développement. Est-il possible d'envisager un développement durable des pays africains si le sentiment d'appartenance des populations aux collectivités nationales et locales continue de se dégrader ?" (1998: 8). 
15 3) La citation d'Elong Mbassi suggère un lien implicite entre l'action de la coopération au développement local et la dégradation de la citoyenneté, qui me permet d'évoquer un dernier point de désaccord avec D. Gentil, celui qui concerne l'interprétation des modalités et des effets de l'action en développement local. Ce point lui tient particulièrement à cœur si j'en juge le nombre de citations de mon article qu'il lui consacre comme autant de preuves de mon forfait. Le lien établi par Elong Mbassi est-il vérifiable? Il n'est certainement pas strictement causal et implique des variables multiples, mais il me semble que sa réflexion touche une question de fond, celle de savoir si les projets peuvent être accusés d'avoir les effets opposés de ceux qu'ils prétendent avoir officiellement. Pour y répondre, nous avons besoin de plus de recherches, à la fois :

- sur la sociologie de l'action en matière de coopération, c'est-à-dire sur la manière dont leurs obligations de résultats (mobiliser les populations, créer des biens publics, motiver les opérateurs économiques tout en continuant à justifier l'utilité de leurs interventions) rendent nécessaires la constitution par les agences de développement, dans leurs rapports au terrain, d'une "économie politique" spécifique, d'une méthodologie ad hoc dont les techniques particulières - on en listera un certain nombre ci-dessous - ont peu à voir avec les grandes valeurs auxquelles elles se référent a priori ;

- sur les effets de composition de l'aide et sur son impact collectif sur les sociétés bénéficiaires, avec l'hypothèse que l'aide vit un dilemme du prisonnier. assez classique, la recherche de l'optimum individuel par chaque intervention et le déploiement de chaque système d'action visant cet optimum donnant un résultat négatif pour tous (Naudet 1999). Les agents de terrain ont une manière particulière de percevoir (et donc de corroborer) l'existence de ce dilemme, lorsqu'ils se plaignent par exemple du manque de coordination, de concertation ou de normes communes entre opérations, cette absence de coordination n'étant pas, pour paraphraser Chauveau (1994 : 52) un "défaut" du système, mais le produit d'un habitus culturel visant sa reproduction en l'état.

16 Sans pouvoir fournir aujourd'hui des analyses très poussées et qui répondraient aux deux pistes de recherche complémentaires que j'ai indiquées, je suis pourtant frappé par la manière dont les fondements de l'action de développement tels qu'ils sont identifiés par les quelques socio-anthropologues qui s'intéressent au sujet - dont nombre de chercheurs apadiens - constituent autant d'éléments d'une "science politique", d'une manière d'imposer sa volonté malgré les résistances, proche dans sa nature des modalités par lesquelles les gouvernants maintiennent leur pouvoir sur les gouvernés : priorité des hommes - les courtiers - sur les institutions, usage de circuits courts, recours au populisme bureaucratique, production d'un champ institutionnel pléthorique, et amnésie permanente vis-à-vis des politiques passées, instauration d'une participation populaire sans droit réel d'opposition, lancement ponctuel de réformes "totalisantes", dosages subtils d'ignorance et de connaissance dans la gestion des projets, absence généralisée d'imputation...

17 Comment dans ces conditions ne pas s'intéresser à des modèles de coopération alternatifs, à "ce qui pourrait arriver" si ces effets de composition étaient pris en compte, si cette sociologie de l'action était critiquée, si une réflexion était maintenue sur les "tiers" (espace public, citoyenneté, ethnicité morale ${ }^{5}$ ?) aptes à rompre l'enfermement du débat actuel sur le développement local entre le "bailleur" et "ses" populations? Etant donnés les atermoiements que je signale et les pressions auxquelles la réforme est soumise, la décentralisation ne contribuera peut-être pas à faire avancer 
de manière décisive la société en la matière. Et pourtant, je ne suis pas le premier chercheur (cf. Fay 1998) à constater que malgré les tensions qu'elles suscitent, la négociation des politiques et leur mise en œuvre (lorsqu'elles ont lieu) sont marquées par une prise en compte du pluralisme des opinions et la contestation croissante du discours technique et du monopole des experts sur les choix de développement opérés. Un large champ d'observation et d'investigation s'ouvre à nous, qui concerne à la fois la recherche théorique et la recherche appliquée. Pour s'y lancer, il faut vigoureusement rejeter l'empirisme idéologique, l'analyse concrète et localisée élevée au rang de leçon normative à laquelle nous invite Dominique Gentil.

\section{BIBLIOGRAPHIE}

Amselle, Jean-Loup. 1998. "Une source majeure de l'idéologie de la décentralisation au Mali : le N'ko". In Pouvoirs locaux. pouvoirs d'Etat. démocratie et décentralisation au Mali, Rapport d'étape. ISH-EHESS-Ministère de la Coopération : 7-23.

Chauveau, Jean-Pierre. 1994. "Participation paysanne et populisme bureaucratique. Essai d'histoire et de sociologie de la culture du développement", In Jacob, J-P, Lavigne Delville, P. (dir), Les associations paysannes en Afrique. Organisation et dynamiques, Paris : Karthala : 25-60.

Elong Mbassi, Jean-Pierre. 1998. "Politiques de décentralisation et projets de développement local", Communication à l'atelier AFD sur les projets de développement local, Paris, 1-4 septembre 1998.

Fay, Claude, 1998. "En attendant les élections". Conclusions provisoires", In Pouvoirs locaux, pouvoirs d'Etat, démocratie et décentralisation au Mali, Rapport d'étape. ISH-EHESS-Ministère de la Coopération : 157-162.

Habermas, Jurgen. 1998.L'intégration républicaine. Essais de théorie politique. Paris : Fayard.

Jacob, Jean-Pierre. 1998a. "L'enlisement des réformes de l'administration locale en milieu rural africain. La difficile négociation de la décision de décentraliser par les Etats et les intervenants externes" Bulletin de l'APAD 15 : 119-137.

Jacob, Jean-Pierre. 1998b. "La décentralisation comme distance. Réflexions sur la mise en place des collectivités territoriales en milieu rural ouest-africain", Politique Africaine 71 : 133-147.

Lonsdale, John. 1996. "Ethnicité morale et tribalisme politique", Politique Africaine 61 : 98-115

Naudet, Jean-David. 1999. Trouver des problèmes aux solutions. 20 ans d'aide au Sahel. Paris : Editions de l'OCDE.

Sartori, Giovanni. 1987. The Theory of Democracy Revisited. Chatham : Chatham House Publishers.

Slater, David. 1990."Debating Decentralization. A Reply to Rondinelli", Development and Change 21(3) : 501-512. 


\section{NOTES}

1.Aucune politique ne pouvant se faire sans se référer au passé, comme le remarque pertinemment J.-L. Amselle (1998), les différents pays proposent des versions "locales" du modèle communautarien. Voir dans ce sens le rôle du N'ko au Mali tel qu'il est analysé par cet auteur.

2.La démocratie représentative n'est sûrement pas le seul système politique possible, comme le souligne Dominique Gentil, mais elle le devient rapidement lorsque les citoyens sont trop nombreux (disons 5.000 personnes) pour être encore capables de décider directement entre eux et qu'ils veulent malgré tout minimiser les risques d'une prise de décision par autrui trop contraire à leurs intérêts.

3."Totales" en ce sens qu'elles se présentent à la fois comme des aides à la constitution de biens matériels et immatériels, et comme des expériences politiques. Encore qu'il me semble intéressant de s'interroger sur les théories politiques sous-jacentes aux instruments de développement mis au point par les experts, mon texte voulait simplement évoquer cette lutte entre deux grandes conceptions que se font partisans du développement local et partisans de la décentralisation. Je reconnais n'avoir aucun droit à classer, comme je l'ai fait dans mon article, Gentil parmi tel ou tel groupe de communautariens, aussi respectables soient leurs inspirateurs (Gandhi, Charles Taylor). Pour cela, je lui adresse mes excuses.

4.D. Gentil confond sujet de l'énoncé et sujet de l'énonciation lorsqu'il prend mes formules qui décrivent la position des décideurs et les dilemmes auxquels ils sont confrontés comme des injonctions personnelles à une mise en œuvre autoritaire des réformes. Sur mon "autoritarisme" et ses justifications théoriques, voir plus loin. 5.Cette référence à l'ethnicité morale montre qu'il est sûrement possible de poursuivre de manière non ethnocentrique cette réflexion sur le tiers. Sur le sujet, voir l'article de J. Lonsdale (1996).

\section{AUTEUR}

\section{JEAN-PIERRE JACOB}

IUED, 24 rue Rothschild, CP 136, CH-1211 Genève (Suisse). Tél. +41 (22) 906 5963. Fax. +41 (22)

9065947.jacob@uni2a.unige.ch 